insuperable, though real, in this hot climate. A good deal can be done to meet them with ice from the local factory; and an efficient cooling plant, specially designed to meet the requirements of an eclipse dark room, should make matters still easier by providing a good supply of cooled water.

Most of the personnel for the actual observations have already arrived, but special mention must be made here of the services to the British expeditions of their honorary secretary, Col. J. Waley Cohen. Not only did he thoroughly explore both sides of the peninsula in 1928, bringing back valuable information as to meteorological conditions and as to local possibilities for eclipse preparations-and incidentally he interested many influential people in the coming eclipse-but he also arrived in the East this year ahead of the observers and made all the preparatory arrangements, so that a great deal was already done and in hand when they arrived. At Pattani, Col. Waley Cohen has also continued to relieve the scientists of the expedition of all worries about such matters as messing, local financial arrangements (not easy when there is no bank within many hours' journey of the camp), and the multitudinous details which have to be attended to, if matters are to go smoothly.

The accompanying photographs, taken by Dr. Royds, director of the Kodaikanal Observatory, show (Fig. 1) the special camp erected for the observers to live in, and (Fig. 2) the astrographic telescope from Greenwich in course of erection with Mr. P. J. Melotte's instruments, including a coronagraph of $19 \mathrm{ft}$. focal length with a direct vision prism for the first and second flash, three spectrographs, and a double tube camera with a Nicol prism in front of one object glass for a polariscopic study of the corona. The party of the observers and assistants on the day of the eclipse will be twelve. In addition to those above mentioned and myself, Prof. E. Barnes and W. F. Kibble, of Madras, have already been at the camp for some days and given valuable help.

\title{
Einstein's and other Unitary Field Theories: An Explanation for the General Reader.
} By Prof. H. T. H. Piaggio.

II.

\section{Geometry on a Sphere.}

$\mathrm{T}$ HE leading ideas of the geometry that Einstein chose (Riemannian) can be made clear by considering the properties of a geographical globe (Fig. 1) on which are marked the meridians and parallels of latitude. These divide the surface into what we may call curvilinear rectangles. But these rectangles are not all of the same size or shape. For consider two points with the same latitude but

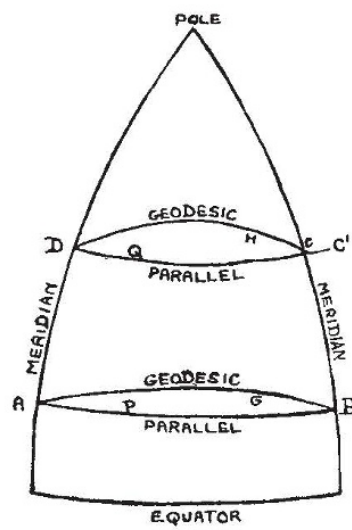

Frg. 1. with longitudes differing by one degree. The distance between them depends upon the latitude; it is greatest at the equator and zero at the poles. Thus APB is greater than DQC. For a sphere the distance between two points with the same longitude (i.e. on the same meridian), but with latitudes differing by one degree, is constant, but if our globe (like the earth itself) is flattened at the poles, this distance will again depend upon the latitude.

In either case, we cannot find the distance between two points $\mathrm{A}$ and $\mathrm{C}$ on the globe merely by knowing the differences of their latitudes and their longitudes, whereas in a plane the distance between two points is determined solely by a knowledge of the differences of their $x$ and of their $y$ co-ordinates. This is what is meant by the rather alarming statement that the sphere has a Riemannian metric, while the plane has a Euclidean one. (In mathematical symbols, $d s^{2}=d x^{2}+d y^{2}$ shows a Euclidean metric, but $d s^{2}=g_{11} d x^{2}+g_{22} d y^{2}$ shows a Riemannian metric, provided that $g_{11}$ and $g_{22}$ are functions of $x$ and $y$, or of either of them.)

It is not only a plane that has a Euclidean metric. Take a piece of squared paper, and roll it up, or bend it (without stretching or tearing) into as queer a shape as you please. The squares drawn on it remain all of the same size as before, hence the metric is still Euclidean. Such a surface is said to have zero Gaussian curvature, although it is what an ordinary person would call curved. The real distinction between it and a sphere is that the squared paper can be flattened out again, whereas it is impossible to flatten out a sphere or a piece of it (as may be easily verified with a piece of a broken rubber ball). Another way of putting this is to say that any attempt to make a flat map of the earth must be imperfect and give a distorted representation, as is obvious on Mercator's chart near the poles.

A well-known problem in geography or navigation is to determine the shortest route that can be traversed between two points on the earth's surface. On a model globe we can determine this experimentally by stretching a piece of string between these two points. It will be found that it will lie in what is called a Great Circle, namely, one the plane of which passes through the centre of the earth. It is important to notice that it is not the same as a parallel of latitude. In fact a ship that has to sail between two points $\mathrm{A}$ and $\mathrm{B}$ on the same parallel APB (north of the equator) will, to follow the Great Circle AGB, have to sail north of this parallel and then come back to it, a method rather tiresome to navigators, as it entails a continual change of direction (as measured by compass bearing). A Great Circle on a sphere has one of the properties of a straight line in a plane, namely, that of being a geodesic or shortest distance between

No. 3110, VoL. 123] 
two points on it, but not (in general) another, that of having a constant direction. This may be considered to belong to a route that makes a constant angle with the meridians; it is called by navigators a rhumb-line or loxodrome. They often use it, in spite of it not being a geodesic, because it preserves a constant compass bearing, which can be determined at once by drawing a straight line between the two positions, as marked on Mercator's chart. It is important to notice that what we here call constant direction on a sphere is defined by reference to compass bearing, or Pole star, or axis of rotation (through which the meridians pass), each of which is really quite independent of the geometry of the sphere itself, and to that extent is arbitrary.

\section{Geometrical Basis of Einstein's} GENERAL THEORY.

Einstein's General Theory may now be stated broadly as the assumption that the physical geometry of space-time is one which has a Riemannian metric and a curvature, and, in fact, is somewhat analogous to geometry on a sphere. The analogy is made closer if we replace the sphere by a surface like a hen's egg, of which the curvature is variable. If the egg has been hard-boiled and then deprived of its shell, so as to be flexible, the analogy is still further improved, for the Gaussian curvature and Riemannian metric, which depend only on a network of curves drawn on the surface and deformable with it, are the properties with which Einstein is concerned. It is important to notice that no account is taken of any measurements except those made on the surface, which from this point of view is a two-dimensional region.

The non-mathematical reader may, however, say, "How can two-dimensional results on a sphere or egg, which everyone can imagine, be applicable to four dimensions, which are inconceivable?" The answer to this is that the symbols used by mathematicians have the valuable property that they enable us to work, largely by analogy, in four dimensions almost as easily as in two. The merit of Riemannian geometry, which to those unfamiliar with it may appear rather complicated, is that in it the physical laws of the motion of a planet or of a ray of light are the simplest possible, namely, that they are geodesics. By stipulating that the paths must be very nearly those given by Newton's law of gravitation, we get some indication of how to determine the coefficients in the Riemannian metric. To determine these fully requires other considerations too lengthy to enter into here.

As is now well known, this theory has been strikingly successful, not only in explaining a known fact, the anomalous motion of the perihelion of the planet Mercury, but also in predicting successfully the effect of a strong gravitational field on the bending of light and the shift of spectral lines. Eclipse expeditions speedily confirmed the first prediction, but the second was originally denied by experimenters. The spectral shift is now admitted to exist, and the minute effects due to the sun have been supplemented by the more easily observed effects due to the dark star of enormous density called the Companion of Sirius.

\section{Physical Basis of the Unitary Field Theories of Weyl, EDdington, and EInstein.}

We have seen that the Special Relativity theory is fundamentally an electromagnetic one, while the General Theory is fundamentally gravitational. After constructing a geometry of space-time, specially chosen so as to explain gravitation in a simple manner, Einstein found that electromagnetism could be fitted into the scheme, but could just as well be left out. Now this is scarcely satisfactory. Gravitation and electromagnetism are both physical phenomena, and why should one be considered as an essential property of space, and the other as only an accident? Was the world constructed solely for the requirements of gravitation, and then part of it let off to electromagnetism as a lodger? The obvious thing seemed to be to modify the Riemannian geometry so that it would serve gravitation and electromagnetism equally well.

\section{Geometrical Basis of Weyl's Unitary Theory (1918).}

Einstein had made gravitation appear as a natural consequence of replacing Euclidean geometry by Riemannian, in which the geodesics lose their property of preserving a fixed direction. Weyl proposed to replace Riemannian geometry by another, in which the idea of length is also given up. In his theory, at any rate in its original form, the length of a rod altered every time it passed round an electric current! This theory certainly gave some interesting mathematics, in which equations of the form of Maxwell's electromagnetic ones made their appearance, but as it has received no experimental confirmation whatever, it need not be considered very seriously.

\section{Eddington's Unitary Theory (1921) as a 'GRAPH'.}

Weyl's geometry, formless as it seemed, still retained one definite property, of which Eddington promptly proceeded to divest it. We shall not enter into details, because Eddington avowedly is not claiming to construct a physical theory, but only an illustration or 'graph', which may be looked upon as a device useful in enabling us to grasp certain mathematical relations. He hoped it might throw light on the nature of the forces which prevent an electron from exploding, but up to the present it does not seem to have done so.

Eddington considers that not only his own unitary theory, but also Weyl's and Einstein's, are 'graphs'. However, from Einstein's own words"my opinion is that our space-time continuum has a structure of the kind here outlined "-it would appear that it is claimed to be a genuine physical theory.

$$
\text { No. 3110, Vor. 123] }
$$


Einstein's Unitary Theory (1928-29).

Whereas Weyl and Eddington replaced Riemannian geometry by others still more unlike Euclidean, Einstein has now, in part, returned to more ordinary ideas. His geometry is one which possesses distant-parallelism as well as a Riemannian metric. To explain what is meant by distantparallelism, we return to our two-dimensional analogy. Cover our hen's egg, or any other surface, with a network of 'curvilinear rectangles'. 'Parallel directions' are defined as those which make the same angles with corresponding sides of the local rectangles. This definition leaves the original choice of the network undefined, but we saw that on a sphere direction had to be defined by something, like a magnetic compass or a pole star, which was not a property of the sphere itself, and so in a certain sense undefined by its geometry alone.

Perhaps Einstein's parallel directions may be ultimately defined in terms of dynamics. He may even get back to the position of Newton, who conceived absolute rotation to be a real thing, which could be detected by seeing whether the surface of a fluid was a paraboloid of revolution or a plane. The behaviour of Foucault's pendulum and of gyroscopes certainly seem to furnish us with a dynamical definition of direction.

By using our sphere, we may even give some idea of the actual function that Einstein takes to measure what may be called electromagnetic potential. Suppose a boat has two short trips, each of one mile, one east and the other north. By sailing first one mile east and then one mile north, let us reach a point $\mathrm{C}$. By sailing first north and then east we reach a different point $\mathrm{C}^{\prime}$, since the parallels of latitude get smaller as we go north (see Fig. 1). The distance $C^{\prime} C^{\prime}$ represents Einstein's potential. This illustration is not exact, because on a sphere $\mathrm{CC}^{\prime}$ is very small compared with the distances $A B, B C$, whereas in Einstein's theory it is essential that it should not be so. To illustrate this we should have to suppose our sphere to have a crinkly surface.

If we now take the corresponding construction for three dimensions, the result is rather queer. If $\mathrm{AB}$ and $\mathrm{DC}$ are 'parallel' paths, the path from $\mathrm{B}$ 'parallel' to $\mathrm{AD}$ will not intersect $\mathrm{DC}$. It is properties of this kind that Eddington finds unattractive, but they are essential to the electromagnetic part of the theory.

Of course the ultimate test of the theory must be by experiment. It may succeed in predicting some interaction between gravitation and electromagnetism which can be confirmed by observation. On the other hand, it may be only a 'graph' and so outside the ken of the ordinary physicist. Einstein's paper points out that so far there has not been time to examine the full consequence of his equations.

Even supposing the theory fully established, there are still fresh worlds for Einstein to conquer. The quantum theory remains outside his scheme. He made an attempt to deal with this so far back as 1923 , but without any striking success. However, it has been suggested that the postulate of distant-parallelism will enable the unitary theory to take over Dirac's theory of a spinning electron almost unchanged.

\section{The Detection of Helium.}

$\mathrm{T}^{\mathrm{H}}$ HE natural facility with which the radioactive elements disintegrate has led on one hand to attempts to break down atoms artificially, and on the other to build them up from simpler particles. Rutherford succeeded in conveying the necessary energy to some of the less massive atoms and broke them down by bombardment with sufficiently energetic $a$-rays, atom by atom at comparatively rare intervals : the process of atom building is still not more than a dream, realised perhaps in the depths of space as Millikan has suggested in order to account for cosmic rays.

The production of gold from mercury, and many another attempted transmutation, have proved, to put it mildly, apparent rather than real changes. In the case of the experiments in which helium was supposedly formed in some way or another by an electric discharge, there has lurked for a long while a certain feeling of unsatisfactoriness. Prof. Paneth's recent work goes far to dispel this feeling (see Zeits. f. phys. Chem., 134, 353; 1928; and $1,170$ and $253 ; 1928)$. The outcome is indeed satisfactory: those that found helium have reason to have got it; those that did not might well have found it, and been misled perhaps as to its origin.

Paneth and Peters show that helium is the only gas which at ordinary temperatures can diffuse through glass. At a pressure of 0.5 atmosphere $10^{-11}$ c.c. of helium will pass through a thickness of $0.5 \mathrm{~mm}$. of soda glass per $\mathrm{cm} .{ }^{2}$ per hour. The amount of helium that gets through from the air at ordinary pressure into an evacuated glass vessel ( $1 \mathrm{~mm}$. wall thickness) is $10^{5}$ times less, so that a glass apparatus is for all practical purposes 'tight ' at ordinary temperatures. When warm the rate of diffusion through the glass is much greater (cf. Lo Surdo, Atti R. Accad. Lincei, 30, 1, 85; 1921). A hard glass tube $1.5 \mathrm{~mm}$. thick at $500^{\circ} \mathrm{C}$. lets through $10^{-9}$ c.c. of helium from the air per em. ${ }^{2}$ per hour. Helium, indeed, can be separated from neon and other gases by diffusion through hot glass. It is otherwise with palladium. Helium will not diffuse through palladium at a red heat. A mixture of helium and hydrogen can be separated completely by diffusion of the hydrogen through a palladium capillary; the quantity of helium that gets through is not even $10^{-12}$ of the quantity of hydrogen that passes. Helium and neon are found in the gases absorbed by glass which has been in contact with air, but the gas is considerably richer in helium than in neon. On the other hand, if there is a minute flaw in the glass or at a tap, causing a leak however small, the neon and helium found in the residual gases remain in the same proportion as

$$
\text { No. 3110, Vol. 123] }
$$

